\title{
RATCHET ANALYSIS OF STRUCTURES UNDER A GENERALISED CYCLIC LOAD HISTORY
}

\author{
Michael Lytwyn \\ Department of Mechanical \& Aerospace Engineering \\ University of Strathclyde \\ Glasgow, Scotland, UK
}

\author{
Haofeng Chen \\ Department of Mechanical \& Aerospace Engineering \\ University of Strathclyde \\ Glasgow, Scotland, UK
}

\author{
Michael Martin \\ Rolls-Royce Power Engineering plc \\ PO Box 2000, Derby, DE21 7XX, UK
}

\begin{abstract}
This paper introduces a new approach based upon the Linear Matching Method in order to obtain the ratchet limit of structures subjected to an arbitrary thermo-mechanical load history. This method varies from the traditional Linear Matching Method ratchet analysis, where the cyclic load history is decomposed into cyclic and constant components, instead calculating the ratchet limit with respect to a proportional cyclic load variation, as opposed to an additional constant load. The shakedown and limit load boundaries are initially obtained for the given structure, followed by the utilisation of a bisection procedure in order to calculate an approximate ratchet boundary based upon a predefined magnitude of ratchet strain per cycle. The method also yields the total and plastic strain ranges based upon perfect plasticity, for low-cycle fatigue post-processing considerations. The effects of analysing the ratcheting mechanism of structures undergoing a cyclic primary load that varies proportionally with a cyclic secondary load can be seen to lead to modified and less conservative ratchet boundaries compared to the traditional Bree solution in which the thermal ratcheting requirement (NB-3222.5) of ASME III is based upon. This paper introduces the theory, numerical implementation and verification of the proposed method via a series of example problems.
\end{abstract}

Keywords: Linear Matching Method (LMM), shakedown, ratcheting, direct method, cyclic plasticity

\section{INTRODUCTION}

Many structural power plant components in engineering experience thermo-mechanical stress histories and as a result may be vulnerable to various cyclic plasticity failure mechanisms. In common nuclear structural integrity codes such as ASME III Subsection NB [1] or the British Energy R5 procedure [2], structures are assessed against the potential for ratcheting failure to occur by means of using stress classification lines (SCL) alongside linearised stresses, relative to specified allowable stress ranges.

There are several failure responses a structure may exhibit under the influence of thermo-mechanical cyclic loading, namely; pure elastic action, strict (elastic) shakedown, global shakedown, ratcheting or plastic collapse (i.e. limit load). The strict shakedown limit is the highest cyclic load under which a material shakes down to a purely elastic response after the first few applications of the load cycle. When the strict shakedown limit is exceeded, the structure may experience either global shakedown or ratcheting. In many applications, it may be deemed overly conservative for a structure to be operating solely within the strict shakedown limit and hence most design codes permit structures to operate within the global shakedown region provided a low cycle fatigue assessment is conducted. Global shakedown, also referred to as plastic shakedown or alternating plasticity, involves a closed loop hysteresis cycle during the steady-state phase, in which plastic straining occurs during each loading/unloading event but no net increment remains. Ratcheting, which ultimately leads to incremental plastic collapse due to the accumulation of plastic strain increments, is usually strictly avoided in order to ensure dimensional integrity of a component throughout its operating lifetime. The high temperatures in power plant components often arise in parallel with high levels of pressure and as such there can often be a direct correlation between the magnitude of primary and secondary stresses throughout the operating cycle [3]. The determination of the ratchet limit via direct methods however is traditionally obtained with respect to an additional constant loading, as originally presented by Miller [4] and Bree [5]. However in reality most industrial power plant applications involve a primary load component, which can then be seen to tend to zero at cold shutdown. The method presented in this paper has been developed with this point in mind; with the primary stress cycling in phase with the secondary stress for the structural problems considered hereafter, which may be deemed 
more realistic than the limiting restrictions arising from the original Bree analysis. This reasoning is one driver behind the development of a direct ratchet analysis tool capable of assessing arbitrary thermo-mechanical load histories, in the sense that over-conservatism may be evident in situations where the original Bree analysis has been used as a Code reference point to adjudge whether or not ratcheting occurs, but in reality the component may still have a significant capacity to accommodate higher levels of cyclic loading before ratcheting occurs. Which in turn relates to the potential to raise the overall plant efficiency as a result of increasing the plant operating conditions. A fundamental issue is that there are currently no plasticity bounding theorems in existence that the Authors are aware of which are capable of directly evaluating arbitrary thermo-mechanical cyclic load histories. Various computational methods have been developed in order to obtain the ratchet limit, including the Linear Matching Method (LMM) [6], Direct Cyclic Analysis (DCA) [7, 8], the Non-Cyclic Method [9], the Elastic Compensation Method (ECM) [10], the Mathematical Programming Method [11] and the Generalized Local Stress Strain (GLOSS) r-node method [12]. The LMM is a direct method which is capable of calculating the ratchet limit via repetitive linear elastic simulations, involving a matching modulus which is used to replicate the actual nonlinear plastic response of a problem both spatially and in time. The fundamental premise of a direct method is to evaluate a scalar load parameter that corresponds to a magnitude of load or temperature history (or both) that is applied to an elastic plastic body so that a predetermined restriction on the deformation history is satisfied. The LMM is differentiated by non direct cyclic methods in the sense that the full load cycle does not need to be fully analysed in a typical calculation, only the relevant peaks in the stress domain which give rise to the largest stress ranges need to be considered. The novel LMM procedure presented in this paper can deliver a scalar parameter that defines the strict shakedown, collapse and ratchet domains via a single calculation. All of the aforementioned computational methods aim to improve ratcheting and shakedown analysis procedures by utilising the latest commercial finite element software as well as moving away from the labour intensive and often subjective stress classification line approaches, which do not always provide adequate solutions [8]. The vast majority of direct methods for ratchet limit detection are based upon the premise of decomposing the general cyclic load history into cyclic and constant components respectively, which inherently means that they are incapable of assessing arbitrary cyclic ratchet limits. The emphasis of this paper is to present a numerical procedure for the evaluation of structures undergoing arbitrary thermomechanical cyclic load histories using the LMM framework. This paper seeks to consider thermo-mechanical load histories which vary proportionally and in-phase throughout the defined load cycle. The result of varying the thermal and mechanical loads in such a way can be seen to lead to less conservative ratchet safety domains compared to the more restrictive Bree load configuration.
The paper is organised as follows, firstly a brief review of the existing LMM framework is presented in order to provide a background to the novel LMM ratchet analysis procedure presented in this paper. Two numerical examples are then analysed in order to verify the proposed methodology. The paper concludes with possible Code considerations for the modified arbitrary load regimes presented and justification for the use of ratchet tolerant design as an effective analysis tool.

\section{NOMENCLATURE}

$\begin{array}{ll}F(x, t) & \begin{array}{l}\text { External forces acting on a body, at position } \mathrm{x} \text { and } \\ \text { time } \mathrm{t}\end{array} \\ P(x, t) & \text { Cyclic component of load history (mechanical load) } \\ \theta(\mathrm{x}, \mathrm{t}) & \text { Cyclic component of load history (thermal) } \\ \lambda & \text { Load parameter } \\ \mathrm{n} & \text { Number of load instances in the cyclic load history } \\ \mathrm{t}_{\mathrm{n}} & \text { Series of time points within the cyclic load history } \\ \mathrm{N} & \text { The total number of time points in the cyclic load } \\ & \text { history } \\ \tilde{\sigma}_{i j} & \text { Stress tensor } \\ \tilde{\sigma}_{i j}(x, t) & \text { Linear elastic stress history } \\ \rho_{i j}(x, t) & \text { The varying residual stress field during the cycle } \\ \tilde{\sigma}_{i j}^{P} & \text { Mechanical load component } \\ \tilde{\sigma}_{i j}^{\theta} & \text { Thermal load component } \\ \tilde{\sigma}_{i j}^{\Delta}(x, t) & \text { Linear elastic stress due to } \tilde{\sigma}_{i j}^{P} \text { and } \tilde{\sigma}_{i j}^{\theta} \\ \bar{\rho}_{i j}(x) & \text { The constant residual stress field } \\ \dot{\varepsilon}_{i j}(t) & \text { Cyclic strain rate }\end{array}$

\section{DEFINITION OF THE CYCLIC PROBLEM}

In order to define the fundamentals of the LMM we may consider an elastic-perfectly plastic body which is subjected to a general cyclic load condition. The body has a volume $\mathrm{V}$ in which a cyclic history of varying temperature $\theta(x, t)$ is imposed alongside a cyclic history of varying surface loads $P(x, t)$ which act upon the body's surface defined as $\mathrm{S}$. A portion of the surface $S$ denoted here as $S_{u}$ is constrained to have a zero displacement rate $\dot{u}=0$. A typical cycle is defined to occur between $0 \leq t \leq \Delta t$. If we consider the problem of a body subjected to external loads, we can reinterpret the applied cyclic load history as;

$$
F(x, t)=\lambda \theta(x, t)+\lambda P(x, t)
$$

where lambda, $\lambda$, is a load parameter and $\theta(x, t)$ and $P(x, t)$ are cyclic histories of temperature and mechanical load respectively, varying with a cycle time $\Delta t$. The subsequent linear elastic stress history can then be noted as $\tilde{\sigma}_{i j}(x, t)$ and can be seen as;

$$
\tilde{\sigma}_{i j}(x, t)=\lambda \tilde{\sigma}_{i j}^{\Delta}(x, t) \text { where } \tilde{\sigma}_{i j}^{\Delta}(x, t)=\tilde{\sigma}_{i j}^{\theta}+\tilde{\sigma}_{i j}^{P}
$$

The load parameter $\lambda$ allows for a whole class of loading histories to be considered, with $\tilde{\sigma}_{i j}^{\Delta}$ representing the varying elastic stresses $\tilde{\sigma}_{i j}^{\theta}+\tilde{\sigma}_{i j}^{P}$, caused by the loads $\theta(x, t)$ and 
$P(x, t)$ respectively. A relevant point to consider is that the stresses and strain increments can be seen to be asymptotic to the cyclic state, where;

$$
\sigma_{i j}(t)=\sigma_{i j}(t+\Delta t) \text { and } \dot{\varepsilon}_{i j}(t)=\dot{\varepsilon}_{i j}(t+\Delta t)
$$

The general form of the stress solution for cyclic problems involving constant and varying residual stress fields is denoted as;

$$
\sigma_{i j}(x, t)=\tilde{\sigma}_{i j}(x, t)+\bar{\rho}_{i j}(x)+\rho_{i j}(x, t)
$$

This general form can interpreted as containing the elastic solution $\tilde{\sigma}_{i j}(x, t)$, a transient solution accumulated up to the beginning of the cycle $\bar{\rho}(x)$ and a residual solution that constitutes the variations within the cycle $\rho(x, t)$. Where $\bar{\rho}(x)$ denotes a constant residual stress field that corresponds to the residual state of stress at the beginning and end of each cycle.

\section{LMM NUMERICAL PROCEDURES}

\section{Previous LMM Ratchet Analysis Procedure}

The previously published LMM analysis for ratchet limit detection with respect to an additional constant loading [6] incorporated a steady state calculation in tandem with an extended shakedown calculation; in the form of a two stage process where the cyclic load history of a problem is decomposed into cyclic and constant components respectively, i.e. by assessing the cyclic plastic strains and residual stresses associated with the cyclic loads in the first stage before proceeding to calculate the ratchet limit with respect to an additional constant load, via an extended upper bound formulation based upon of Koiter's theorem [13] in the second stage. The limitations of this procedure can be seen to arise mainly from the fact that the method is inherently restricted to Bree type loading regimes, due to the decomposition of the cyclic load history into separate cyclic and constant components, as no plasticity bounding theorems currently exist that are capable of directly evaluating proportional thermomechanical load histories. The method presented in this paper utilises the LMM Direct Steady State Cyclic Analysis (DSSCA) procedure [6], but with a fundamental adaptation in order to tackle the modified thermo-mechanical load regime. The purpose of modifying the DSSCA procedure is to provide an initial attempt at calculating ratchet limits with respect to proportional cyclic load variations, in terms of a predefined magnitude of ratchet strain per cycle. The previously published two stage LMM ratchet strategy is deemed as a well established numerical analysis tool and has been extensively applied to several practical industrial problems [6]. The numerical scheme for LMM ratchet analysis with respect to an additional constant loading using a two stage procedure can be summarised as;

i. $\quad$ Stage 1: Calculation of the varying plastic strains and residual stresses associated with a fixed magnitude of cyclic loading. ii. $\quad$ Stage 2: Calculation of a constant residual stress field and upper bound multiplier via a modified shakedown analysis, using the varying residual stresses from Stage 1 to augment each elastic solution as an input, i.e. $\tilde{\sigma}_{i j}^{\Delta}\left(x, t_{n}\right)+\rho_{i j}(x, t)$.

\section{Ratchet Analysis using a LMM Bisection Procedure}

The method presented in this paper utilises the DSSCA from [6] procedure, but with a fundamental adaptation in order to tackle the modified thermo-mechanical load regime. The method presented in this paper aims to calculate the ratchet limit with respect to a proportional variation between the primary and secondary cyclic loads, with the derived ratchet limit being defined in terms of a predefined magnitude of equivalent ratchet strain per cycle. This infers the use of ratchet tolerant design for assessing structural problems, which even though is strictly prohibited in certain design codes, does however allow for highly accurate approximate ratchet limits to be rapidly produced for structures undergoing realistic cyclic transient loads, which in turn provides the assessor with a strong indication as to where the exact ratchet limit actually lies for a given load cycle. The method is verified using the Bree cylinder with the analytical solutions of Bradford [3] as a reference point, as well as the holed plate structure which is used to investigate multi-axial 3D effects. In order to analyse a typical load cycle, a series of elastic solutions are generated at discrete time points within the load cycle (for example at $n$ locations in time, giving a series of elastic stresses, $\left.\tilde{\sigma}_{i j}^{\Delta}\left(t_{n}\right)\right)$, such that the most significant stress ranges in the load cycle are encapsulated and hence the most significant plastic strains are used to correctly identify and calculate the ratchet mechanism caused by a given load cycle. By doing so, all other time points within the cycle are deemed innocuous in terms of significant plastic straining and are assumed to lie within the von Mises yield surface, thus allowing vast computational efficiency to be gained over conventional CCA methods, which involve analysing the entire load cycle. The LMM presented in this paper is implemented using the UMAT and URDFIL subroutines in ABAQUS [11]. A novel bisection procedure is used for convergence purposes for the method, which is based upon a predefined magnitude of maximum equivalent ratchet strain per cycle (that is stipulated $a$ priori by the analyst). The bisection procedure presented in this paper is capable of obtaining the shakedown, ratchet and limit load regions during one single calculation, hence providing a comprehensive shakedown assessment of the structure. The bisection procedure commences by calculating the strict shakedown limit for a given set of discrete elastic stresses $\tilde{\sigma}_{i j}^{\Delta}\left(t_{n}\right)$ (Step 1), before proceeding to calculate the limit load based upon these linear elastic input stress fields (Step 2). When multiple time points are used to generate the elastic stress fields that represent the load cycle under consideration, then the shakedown and limit loads will naturally be based upon the largest elastic stress range. Once the strict shakedown and limit load multipliers have been obtained, the process then utilises the augmented 
DSSCA methodology in order to calculate the steady-state solution, i.e. the varying plastic and ratchet strains for a given set of elastic stress fields (Step 3). The DSSCA procedure is modified in such a way that Step 3 operates in an iterative manner; whereby the elastic stress fields are repeatedly scaled in order to calculate the plastic strain history throughout the cycle, as opposed to considering a fixed cyclic stress range as in [6]. For example, in order to commence Step 3 of the process, the first bisection multiplier will lie between the strict shakedown multiplier $\lambda_{S H}$ and the limit load multiplier $\lambda_{\text {LIMIT }}$. The user must specify the amount of iterations that will be used in order to calculate the varying plastic strains during each repetition of Step 3, defined here as a sub-cycle. The maximum von Mises equivalent ratchet strain associated with the scaled elastic stresses will then be obtained after each complete subcycle. Convergence of the method is based upon a pre-defined target magnitude of maximum equivalent ratchet strain per cycle; i.e. once a sub-cycle has been completed the maximum equivalent ratchet strain for the structure is obtained, before proceeding to use URDFIL in order to compare this value of equivalent ratchet strain against the pre-defined magnitude and hence a new load multiplier is created, using a bisection of the last two previous load multipliers. Because the numerical procedure presented calculates the strict shakedown limit and limit loads, with various levels of loading also calculated in between these respective limits in the bisection phase of Step 3, the method can also post-process the maximum plastic and total strain ranges associated with each of these various levels of loading and subsequently arrange these strain ranges relative to ascending load levels for low cycle fatigue analysis purposes. The numerical strategy employed for analysing proportional cyclic load histories using the bisection procedure can be summarised as follows;

- Step 1 - Obtain the strict shakedown limit using the upper bound LMM shakedown theorem.

- $\quad$ Step 2 - Obtain the limit load as a special case of the upper bound shakedown theorem, i.e. by using a single time point in the calculation.

- $\quad$ Step 3 - The proportional ratchet limit multiplier is obtained using the novel LMM bisection procedure. The relevant strain ranges from the steady-state cyclic analysis are also attained.

The method is verified using the Bree cylinder with the analytical solutions of Bradford [3] as a reference point, as well as the typical holed plate example. Traditional CCA is used for verification for the holed plate problem, with perfect-plasticity adopted as is standard. Incremental finite element calculations typically offer the ability to generate benchmark numerical results for verification purposes, as such methods possess the ability to analyse any type of load cycle however these methods are often computationally expensive, especially for complex 3D models.

\section{NUMERICAL EXAMPLES}

\section{Bree Cylinder}

The traditional Bree cylinder provides a simple uniaxial demonstration of the method, as well as allowing for direct comparisons to be made with published analytical results for verification purposes [3]. The plane stress Bree cylinder case has been illustrated in Fig. 1, with the problem representative of the fuel clad in a fast reactor configuration [5]. A cyclic thermal load was applied to the inner surface of the cylinder, alongside a cyclic internal pressure. The applied cyclic load history is depicted in Fig. 2, where both the thermal and mechanical loads can be seen to vary in-phase with one another, thus the problem may be characterised by two load extremes; on-load ( $\sigma_{p}$ and $\Delta \theta$ ) and off-load (where both loads are simultaneously removed to a zero stress state). The model was constrained vertically at one end and allowed to expand in-plane at the other, with a thrust applied to the free end to simulate the closed-end condition. The following temperature independent material properties were used in the analysis: thermal conductivity = $0.0215 \mathrm{~W} / \mathrm{mm}^{\circ} \mathrm{C}$, Young's Modulus $=184 \mathrm{GPa}$, Poisson's ratio $=0.3$, coefficient of thermal expansion $=1.84 \mathrm{e}^{-5 \circ} \mathrm{C}^{-1}$ and yield strength $=205 \mathrm{MPa}$. In Fig. 3, the full cyclic interaction diagram for the Bree problem has been derived, using the three step LMM bisection procedure discussed above, which clearly shows the relevant regions for each of the cyclic failure mechanisms. The strict shakedown and collapse limits are exact, as these are obtained using the upper bound LMM shakedown procedure, whilst the ratchet limit is approximately derived for a target magnitude of ratchet strain per cycle equal to $0.02 \%$. Several approximate ratchet limits found using the bisection method can also be seen in Fig. 4, where the abscissa represents the cyclic mechanical load and the ordinate displays the cyclic thermal load as normal, with both axes being normalised against the relative yield strength of the material. The analytical solutions provided by Bradford [3] can be seen in Fig. 4 alongside the ratchet boundaries obtained by using the bisection method with various values of maximum equivalent ratchet strain per cycle, including $0.02 \%, 0.03 \%$ and $0.05 \%$. For this problem, 20 iterations per load instance was used per sub-cycle (meaning that each sub-cycle contained 40 iterations for the two load instances modelled) in order to ensure adequate stress redistribution using the linear matching procedure in the steady cycle analysis. The FE model is constructed using plane stress conditions in order to generate comparison results with Bradford [3]. 


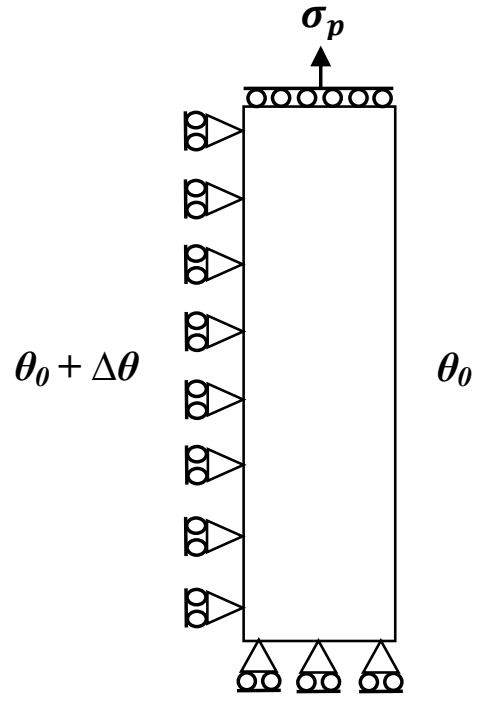

Fig. 1 - Plane stress Bree model

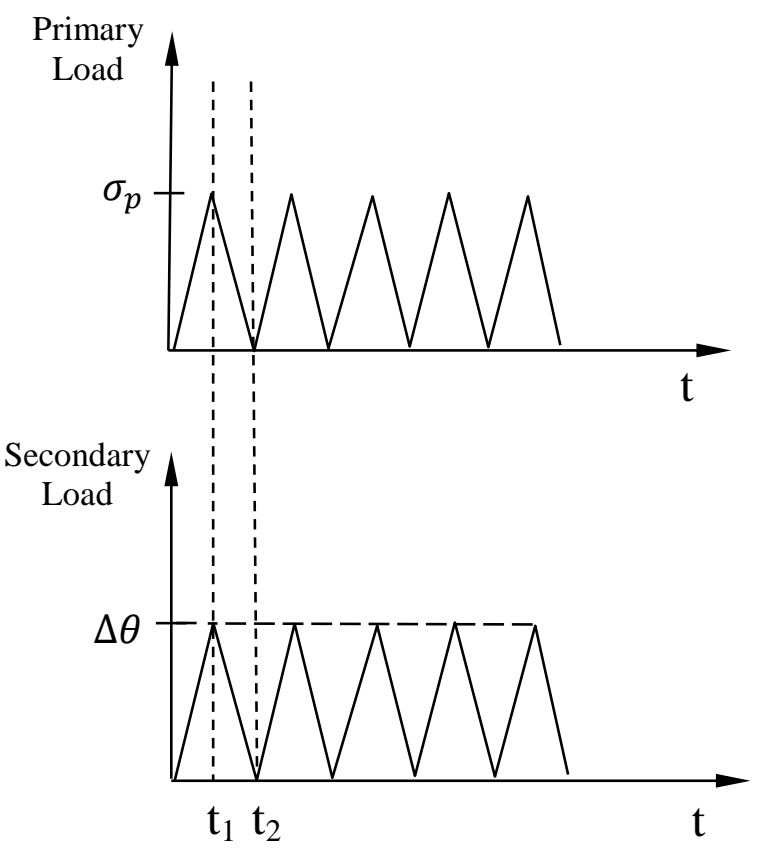

Fig. 2 - Applied proportional cyclic thermo-mechanical load history.

A typical calculation using the bisection method can be seen in Fig. 3 for clarity with an example reference load point shown which constitutes the loaded time point 1 in the calculation (with time point 2 being at zero, i.e. both loads fully removed) before proceeding to the three step bisection procedure; with the entire interaction plot generated via a series of such calculations using varying load paths. The ASME III Code $3 \mathrm{Sm}$ safety envelopes for i) normal Bree type loading and ii) the proportional load case can be seen in Fig. 4, which clearly illustrate the inherent conservatism in the proportional case and the subsequent extra safety margin which is available when both loads vary strictly in-phase $[1,16]$. As is depicted in Fig.
4, the differences between the ratcheting limits are more pronounced as the range of cyclic thermal load increases, with the $0.02 \% / c y c l e$ ratchet limit closely matching the exact analytical solution due to the small extent of the target ratcheting measure used.

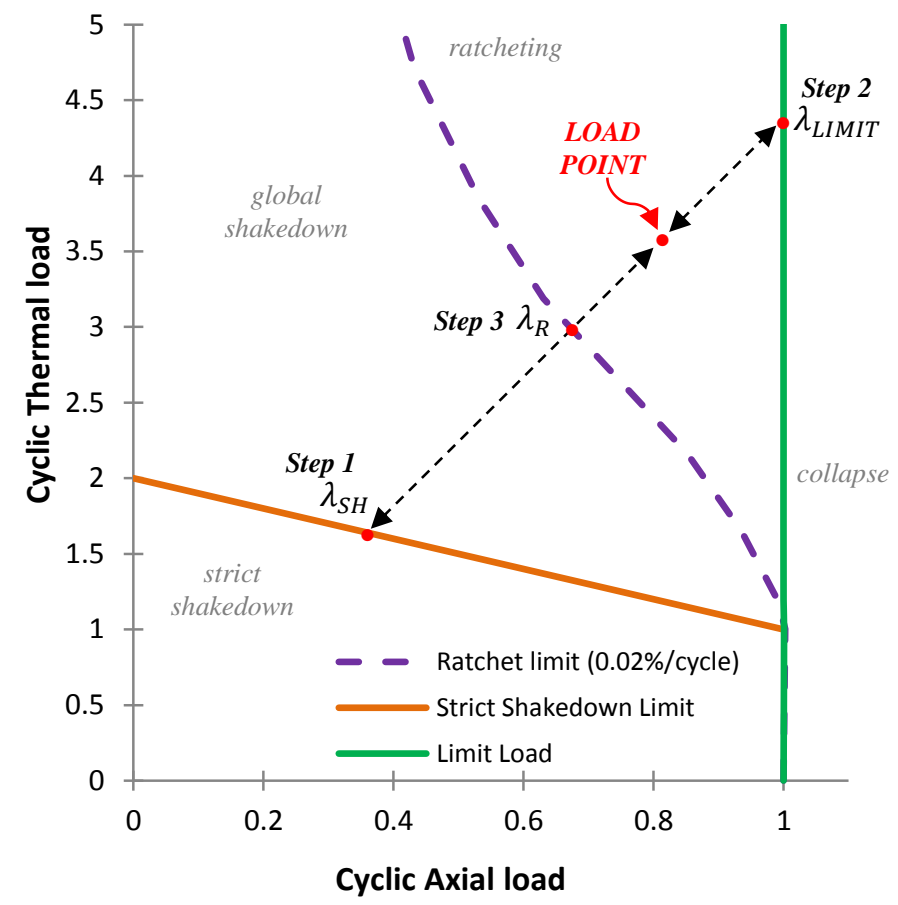

Fig. 3 - Cyclic plasticity limits for the proportional Bree case

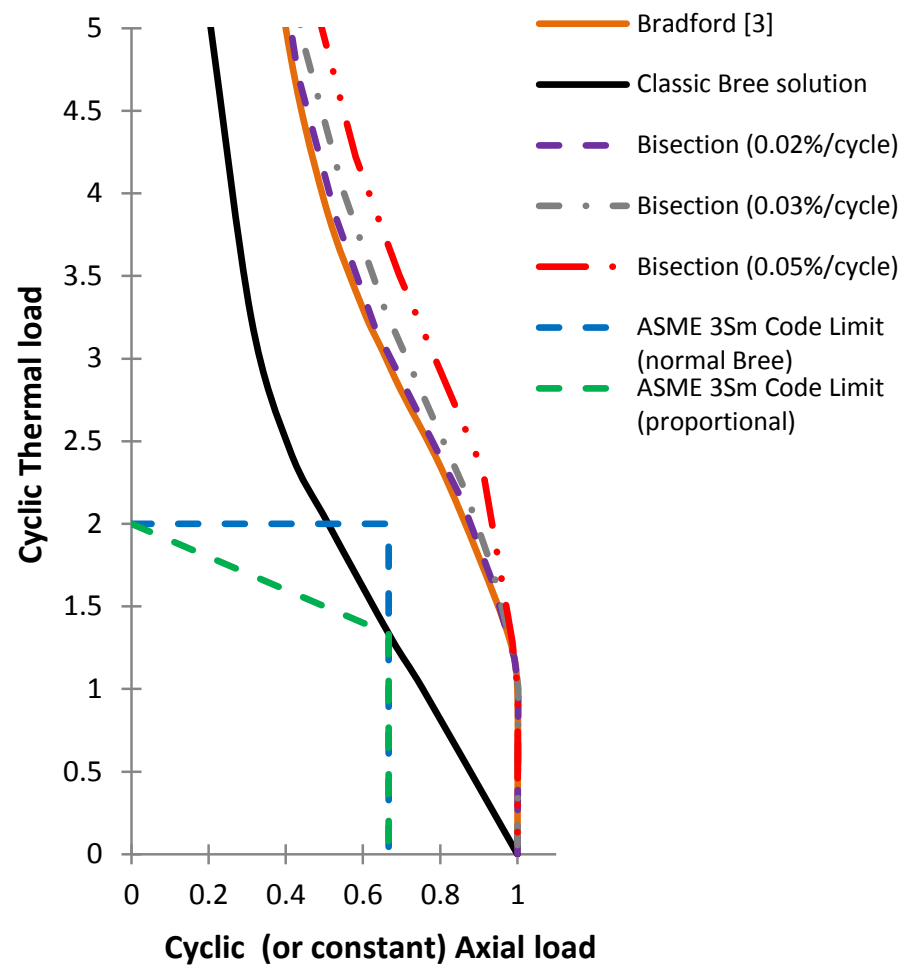

Fig. 4 - Ratchet limits for the proportional Bree case 
A typical display of the convergence characteristics of the three step bisection procedure can be seen in Fig. 5. The process commences from the reference load point shown in Fig. 3, i.e. at $\lambda=1$. Fig. 5 shows the initial strict shakedown and limit load multipliers which are obtained in Steps $1 \& 2$ respectively, before proceeding to illustrate the bisection load multiplier convergence scheme in order to obtain the approximate ratchet limit relative to the predefined target magnitude of ratcheting strain per cycle. As previously stated, the bisection procedure can post-process the maximum equivalent plastic strain ranges (and total equivalent strain ranges) relative to the bisection load multipliers, with an illustration of the variation in these strain ranges shown in Fig. 6 for a series of load levels above the ratchet limit.

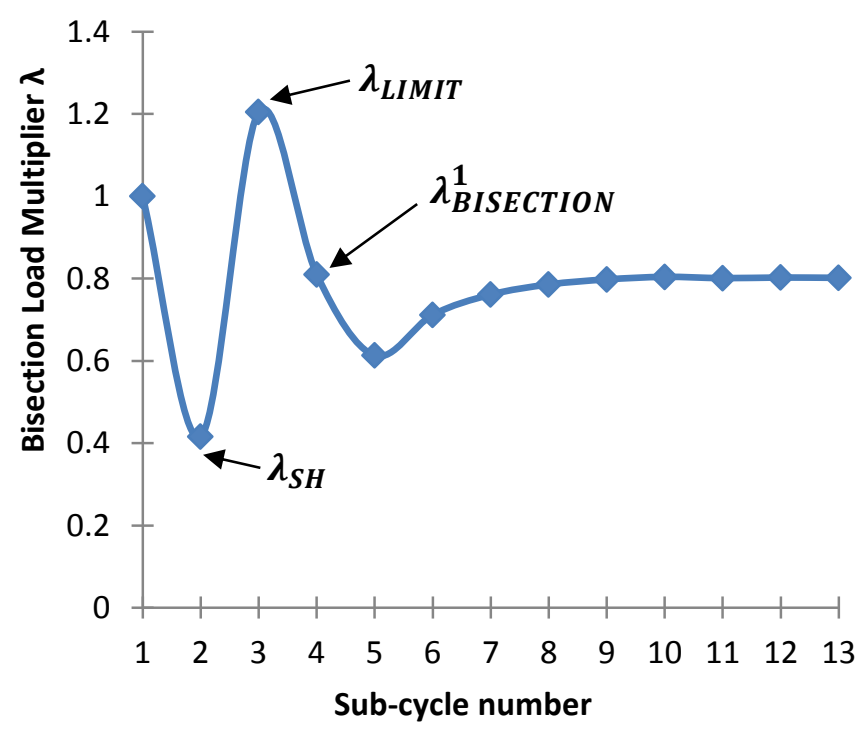

Fig. 5 - Bisection load multiplier convergence

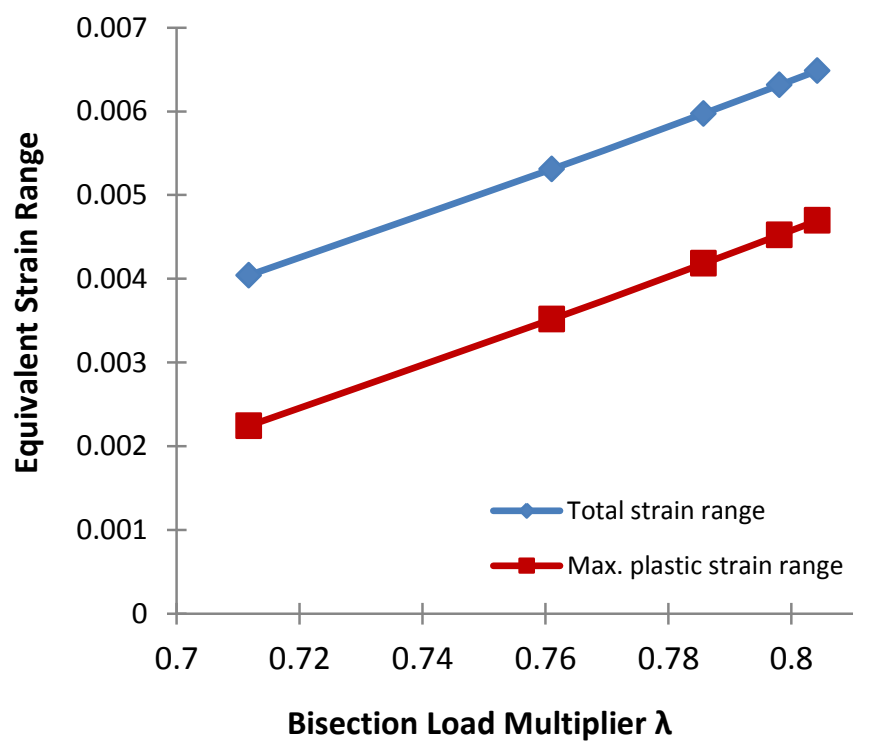

Fig. 6 -Equivalent strain ranges vs. load multiplier

\section{Holed Plate Example}

The holed plate problem provides a 3D example in order to highlight the varying ratchet limits obtained by using a proportional load regime compared to that of an additional constant loading. Ratchet limits derived on the typical combined action of cyclic thermal loading plus an additional constant load component provide a complimentary background to the modified load histories discussed in this paper. Hence this numerical example will use results obtained from the two stage LMM procedure previously quoted in [6], for the additional constant load scenario, in order to provide a comparison with the modified proportional load case. The problem consists of an applied temperature distribution $\Delta \theta$ at the edge of the hole radius, in tandem with a uniaxial tension $\mathrm{P}$ applied on opposite edges of the plate, as portrayed in Fig. 7. The FE mesh used for the analysis is shown in Fig. 8. The holed plate has the same dimensions as used in [6], with the ratio between the diameter $D$ of the hole and the length $L$ of the plate equaling 0.2 , with the ratio of the depth of the plate to the length $L$ of the plate is 0.05 . The temperature-independent material data for the holed plate include; a yield stress $\sigma_{y}=$ $360 \mathrm{MPa}$, elastic modulus $\mathrm{E}=208 \mathrm{GPa}$, Poisson's ratio $v=0.3$ and a coefficient of thermal expansion equal to $5 \times 10^{-5}{ }^{\circ} \mathrm{C}^{-1}$. A quarter model of the plate is used for the analysis due to symmetry conditions, with 20-node quadratic brick elements (ABAQUS C3D20R) used for the structural analysis. A thermal analysis is conducted with $\Delta \theta=100^{\circ} \mathrm{C}$ at the edge of the plate alongside the inner bore of the hole remaining at a constant $\theta_{0}=0^{\circ} \mathrm{C}$ (using DC3D20). The maximum thermo elastic von Mises effective stress occurs at the edge of the hole, which is governed by the applied temperature difference $\Delta \theta$. Hence the extremes of the load history are characterised by $\sigma_{P}$ and $\Delta \theta$ similar to the Bree problem, as depicted in Fig. 2, i.e. the temperature around the edge of the hole varies between 0 and $\Delta \theta$.

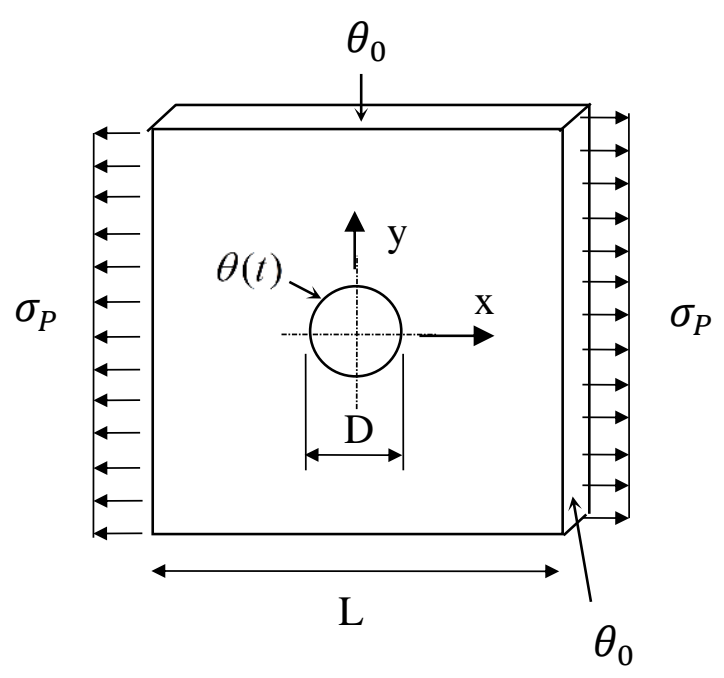

Fig. 7 - Holed plate problem details 


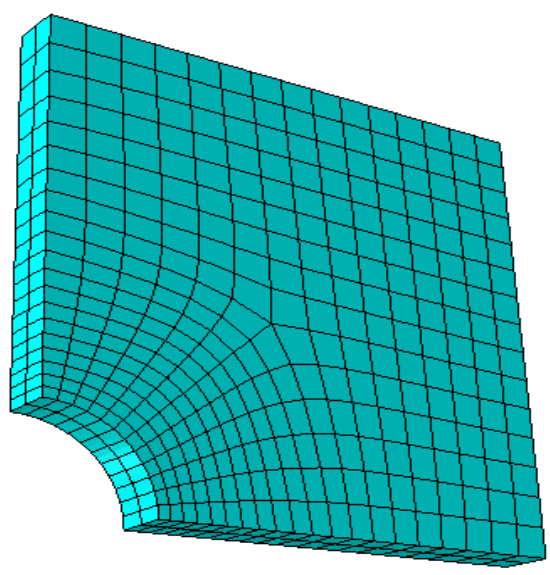

Fig. 8 - Holed plate FE mesh

In Fig. 9, two approximate ratchet limits obtained from the bisection method are derived for $0.04 \%$ and $0.1 \%$ magnitudes of maximum equivalent ratchet strain per cycle respectively. The strict shakedown limit which is derived from a proportional cyclic history (shown as 'Proportional RP limit' in Fig. 9) is also shown in order to highlight the difference in the strict shakedown regions for the typical Bree load regime versus the proportional load case.

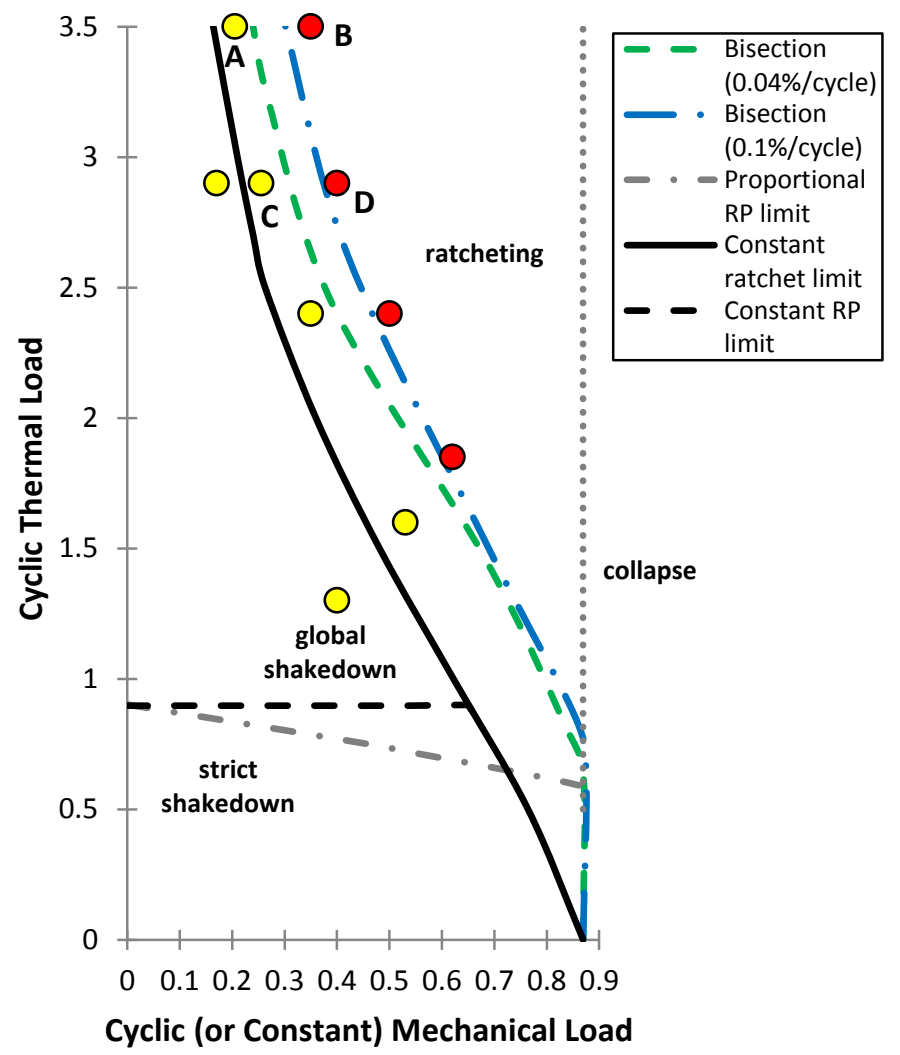

Fig. 9 - Ratchet limits for the proportional holed plate problem
In order to verify the ratchet limits provided by the bisection method and to provide guidance towards the locations of the relative cyclic plasticity failure regions, several CCA calculations were conducted. The yellow markers in Fig. 9 indicate the individual CCA calculations that were found to be in global shakedown and conversely the red markers indicate the individual ratcheting locations. Details relating to the magnitudes of plastic strain from locations A, B, C and D (from Fig. 9) for 250 applied load cycles, using an elastic-perfectly plastic material model, can be seen in Fig. 10. These results illustrate that points A \& C exhibit global shakedown whilst points B \& D display ratcheting behaviour.

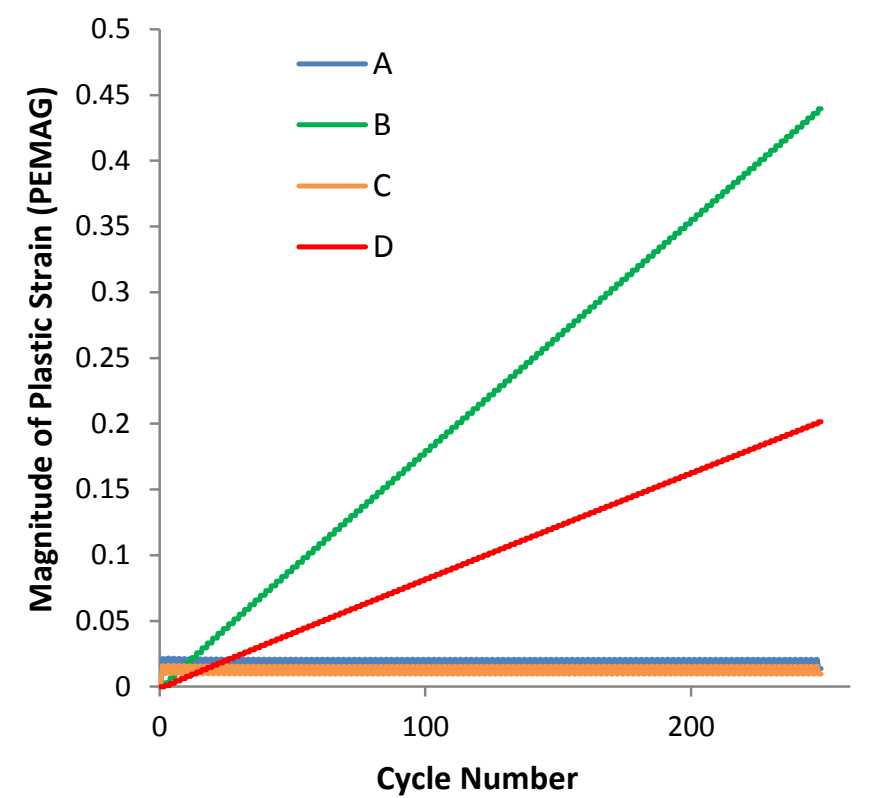

Fig. 10 - Magnitude of plastic strain results from locations A, $\mathrm{B}, \mathrm{C} \& \mathrm{D}$, illustrating ratcheting at B \& D and global shakedown at A \& $\mathrm{C}$.

There are two parameters of the bisection procedure that may be pre-adjusted by the analyst, namely the amount of iterations per sub-cycle and the desired target magnitude of maximum equivalent ratchet strain. As an indicative value, 70 iterations is used per load instance in the holed plate example, which means that the corresponding total amount of iterations per sub-cycle equals $70 \mathrm{x}$ number of elastic load points $=140$ iterations (as two load extremes have been used to represent the applied load cycle). The correlation between stress redistribution and the relative complexity of the finite element model used go in hand in hand with the amount of linear matching iterations needed for accurate solutions to be generated per sub-cycle, highlighted by the Bree example which needs a comparably small amount of iterations per sub-cycle in order to achieve accurate solutions, due to the predominantly uniaxial nature of the problem. 


\section{DISCUSSION}

One of the most fundamental aspects of the bisection method from an implementation aspect arises due to the total number of iterations used per sub-cycle, as this value dictates the relative efficiency and accuracy of the method. Using a larger number of iterations per sub-cycle leads to higher accuracy in the residual stress fields being generated, at the cost of computational expense however. Even though the method may need to involve a large amount of iterations per sub-cycle in order to obtain accurate solutions, it is worthwhile to note that the full cyclic solution for the problem at hand will be obtained as a result, namely the strict shakedown limit, the collapse load, the ratchet limit, as well details of the plastic and total strain ranges between the strict shakedown and limit loads; offering a comprehensive analysis and basis for further LCF calculations. In the example of the Bree cylinder, there is no need to conduct any CCA calculations for verification purposes as analytical solutions based upon plane stress conditions already exist. The analytical solutions (provided by Bradford [3]) are based upon plane stress to allow the solution to be analytically tractable. The basic uniaxial plane stress Bree model still provides a useful example in displaying the relevant plasticity mechanisms, but does not serve as a fully robust test of the proposed numerical method due to the uniaxial nature of the ratcheting mechanism in this case.

The holed plate problem has been used to increase the complexity from the simple uniaxial Bree case, as well as reinforcing the apparent conservatism that is achievable when a proportional cyclic load regime is used, as opposed to the more restrictive constant load ratchet limit.

More complex 3D industrial problems are required to fully evaluate the proposed bisection method, where verification analyses using methods such as CCA are not straightforward due various reasons such as, the availability of material data (especially if temperature dependency is considered), accurate details of the load history may not be fully known, numerical errors may also arise due to the assessment point being on the interface between two different failure mechanisms for example and also interpretation of results based upon the assessor's experience may be an important practical issue. Even though the ASME III Code does not permit ratcheting in a structure operating under cyclic loading conditions, the bisection method still allows for accurate ratchet limit diagrams to be directly obtained by using elements of the LMM framework in order to construct a suitable solution, whilst bypassing the difficulties associated with the modified upper bound theorem of Koiter and arbitrary thermo-mechanical load histories. However, further justification for using a ratchet tolerant tool as such may be found during the design or analysis stage where a shakedown screen needs to be rapidly generated. The bisection method is also relevant when a particular component is subjected to limited load cycles during the overall operating lifetime period, as is commonly found in civil nuclear reactors for example. An example of such behaviour may involve a plant component experiencing a maximum equivalent ratcheting strain per cycle equal to $0.01 \%$ under a given transient. If the total lifetime of the component involved 80 load cycles (assuming this transient is the most severe case in the operating period), due to reactor start up and shut down events, this would still constitute an overall inelastic strain accumulation of only $0.8 \%$ over the entire life of the component. ASME III Section NB-3228.4 states that if a material's minimum yield strength to minimum ultimate strength ratio is less than 0.7 (showing sufficient strain hardening potential) then the shakedown requirement need not be satisfied provided the maximum accumulated local strain limit at any point may is no greater than 5\% [1]. A relevant measure to compliment the ratchet tolerant design concept used in this paper stems from the JPVRC ratcheting check [15], which involves ensuring that the equivalent plastic strain increments at all time points in the load cycle are displaying a decreasing trend whilst the maximum value of the increments is less than $0.01 \%$. Around 5 to 10 cycles is suggested in order to obtain this $0.01 \%$ magnitude for practical purposes $[15,16]$. As shown in Fig. 4 the ASME shakedown safety envelope (as per NB-3222.5) is altered for the proportional load case (due to the primary stress having a range) which infers that the secondary stress range is not independent of the primary stress for this case, as is the case with the original Bree analysis. As is evident from Fig. 4, an in-phase variation of the primary stress with the thermal stress results in a ratchet limit which is notably less conservative than the original Bree analysis. This point is further substantiated by the perceptible difference in the holed plate ratchet limits for the varying load regimes presented. The most apparent change in the ASME Code limits is that the allowable secondary stress range alters from $2 \sigma_{y}$, for the original constant Bree problem, to $4 / 3 \sigma_{y}$ at the primary stress range limit of $2 / 3 \sigma_{y}$ for the proportional load case. This modified Code limit for the strict shakedown region corresponds exactly with the analytical solution derived by Bradford [3] and with the numerical results obtained by the LMM in this paper. Hence the importance of phase consideration between primary and secondary loads is significant, as in realistic power plant scenarios the in-phase load variation may be closer to reality but with the possibility of a small degree of phase difference, i.e. thermal transient stresses in start-up may peak before the pressure fully reaches its full operating value [3].

\section{CONCLUSIONS}

A novel procedure for evaluating proportional thermomechanical cyclic load histories has been presented, evaluated and verified in this paper using the LMM framework. The method is based upon the direct steady state analysis of the LMM procedure and in tandem with a bisection convergence strategy can obtain the strict shakedown, limit load and ratchet limits via a single calculation. The method also yields the cyclic stresses, residual stresses, plastic strain ranges (for the low cycle fatigue assessments) and total strain ranges for various levels of cyclic loading. The numerical procedure presented is used to overcome the difficulties associated with 
previously published extended upper bound theorems of Koiter, as no such bounding theorems currently exist capable of assessing arbitrary load variations, whilst providing results which are deemed as suitable engineering. In order to assess the applicability of the method, two simple benchmark cases have been used to illustrate the numerical procedure, in the form of the Bree cylinder and the holed plate problem. The development of a direct shakedown method for proportional thermo-mechanical loading opens up possible areas of future work associated with the incorporation of more realistic material constitutive models that can account for hardening effects in ratcheting analysis. Proportional methods allow for more realistic descriptions of the plastic strain histories to be attained for a given problem compared to methods which decompose the cyclic solution into constant and cyclic components, thus opening up future research avenues in cyclic material hardening. The Code considerations discussed are reliant on which loads actually cycle in a given transient and the relative phase considerations between primary and secondary loads, a matter which will be evaluated in a more indepth manner as a part of a future work package.

\section{ACKNOWLEGEMENTS}

The authors gratefully acknowledge the Engineering and Physical Sciences Research Council (EPSRC) of the United Kingdom, Rolls-Royce Power Engineering plc. and the University of Strathclyde for their support during this work.

\section{REFERENCES}

[1] ASME Boiler and Pressure Vessel Code, Section III. Rules for construction of nuclear facility components, Division 1subsection NB, class 1 components.

[2] R5, Assessment procedure for the high temperature response of structures, R5 Issue 3, June 2003, EDF Energy.

[3] R. A. W. Bradford, The Bree problem with primary load cycling in-phase with the secondary load, International Journal of Pressure Vessels and Piping, vol. 99-100, pp. 44-50, Nov. 2012.

[4] Miller, D.R, Thermal-stress ratchet mechanism in pressure vessels, Journal of Basic Engineering, Transactions of ASME, pp.190-196, 1959.

[5] Bree J, Elasto-plastic behaviour of thin tubes subjected to internal pressure and intermittent heat fluxes with application to fast reactor fuel elements. Journal Strain Analysis, 196712 , 226-238.

[6] Chen H.F. A Direct Method on the Evaluation of Ratchet Limit, Journal of Pressure Vessel Technology; 132, 2010.

[7] Nguyen-Tajan et al, Determination of the stabilized response of a structure undergoing cyclic thermal-mechanical loads by a direct cyclic method. Abaqus Users' Conference Proceedings, 2003.

[8] Martin, M. Application of direct cyclic analysis to the prediction of plastic shakedown of nuclear power plant components. Pressure Vessels and Piping, Chicago, Illinois, 2008 .
[9] R. Adibi-Asl, W. Reinhardt, Non-cyclic shakedown/ratcheting boundary determination - Part 1: Analytical approach, International Journal of Pressure Vessels and Piping, vol. 88, no. 8-9, pp. 311-320, Aug. 2011.

[10] D. Mackenzie, J. T. Boyle, R. Hamilton, The elastic compensation method for limit and shakedown analysis: a review, The Journal of Strain Analysis for Engineering Design, vol. 35, no. 3, pp. 171-188, Jan. 2000.

[11] D. K. Vu, A.M. Yan, H. Nguyen-Dang, A primal-dual algorithm for shakedown analysis of structures, Computer Methods in Applied Mechanics and Engineering, vol. 193, no. 42-44, pp. 4663-4674, Oct. 2004.

[12] Seshadri R, The generalised local stress strain (GLOSS) analysis - theory and application. Trans ASME, Journal Pressure Vessel Technology;113: 219-227, 1991.

[13] Koiter WT, General theorems for elastic plastic solids. Progress in solid mechanics J.N. Sneddon and R. Hill, eds. North Holland, Amsterdam, 1, 1960;167-221.

[14] W. Reinhardt, Distinguishing ratcheting and shakedown conditions in pressure vessels, ASME PVP Conference, Cleveland, Ohio, USA, 2003.

[15] Okamoto, A., Nishiguchi, I., and Aoki, M., New Secondary Stress Evaluation Criteria Suitable for Finite Element Analyses, ICPVT-9, Sydney, Vol. 2, pp. 613-620, 2000.

[16] Kalnins, A. Shakedown and Ratcheting Directives of ASME BP\&V Code and their Execution. PVP Vol. 439, ASME PVP Conference, Vancouver Canada, pp. 47-55, 2002. 